\title{
Commentaries
}

\section{Double reflux: double trouble}

The noxious agents responsible for injuring the oesophageal mucosa in patients with gastro-oesophageal reflux disease (GORD) may originate from two possible sources, the stomach and the duodenum. Hydrochloric acid and pepsin are the important gastric contents, whereas conjugated and unconjugated bile acids and trypsin are the proposed duodenal ingredients predisposing to the development of oesophageal symptoms and mucosal injury. ${ }^{1}$ The regurgitation of these duodenal contents into the stomach followed by reflux into the oesophagus is known as duodenogastro-oesophageal reflux (DGOR).

To date, the controversy in the literature regarding the specific agents responsible for oesophageal damage centres around the relative importance of acid/pepsin reflux versus DGOR. Early animal studies have clearly shown that acid alone, and in combination with various pepsin concentrations, is damaging to the oesophagus. ${ }^{2-5}$ For example, Goldberg and colleagues ${ }^{2}$ showed oesophageal mucosal damage in the intact feline oesophagus with either very high acid concentrations $(\mathrm{pH} 1.0-1.3)$ or lower acid concentrations $(\mathrm{pH}$ 1.6-2.0) in the presence of pepsin. Additionally, human studies ${ }^{3-5}$ have clearly delineated a positive correlation between the degree of abnormal acid reflux and the severity of oesophagitis. These studies show that more than $90 \%$ of patients with oesophagitis and Barrett's oesophagus have increased oesophageal exposure to acid on $\mathrm{pH}$ monitoring. ${ }^{5}$ Additionally, Bremner and colleagues ${ }^{6}$ observed that patients with increased oesophageal exposure to $\mathrm{pH} 1-2$, corresponding to the known $\mathrm{pKa}$ of pepsin, had the most significant degrees of oesophagitis; an indirect inference to the possible importance of pepsin. Therefore, the role of acid and pepsin in causing oesophageal mucosal injury is irrefutable.

The role of duodenal contents in the development of oesophageal mucosal injury is controversial and the subject of many animal and human studies. Animal studies show that oesophageal mucosal damage caused by bile acids is dependent on both the conjugation state of bile acids and the $\mathrm{pH}$ of the refluxate. Using net acid flux (NAF) across the oesophageal lumen as an index of mucosal injury, Harmon and colleagues ${ }^{7}$ showed that taurine conjugated bile salts, taurodeoxycholate and taurocholate, increased NAF at $\mathrm{pH}$ 2, whereas the unconjugated forms increased $\mathrm{NAF}$ at $\mathrm{pH} 7$ and not at $\mathrm{pH} 2$. Hence, animal studies show that conjugated bile acids are more injurious to the oesophageal mucosa at acidic $\mathrm{pH}$, whereas unconjugated bile acids are more harmful at $\mathrm{pH} 5-8$. Additionally, Kivilaakso and colleagues ${ }^{8}$ found that in addition to bile acids, the pancreatic enzyme trypsin causes oesophageal mucosal damage at $\mathrm{pH}$ 7.0.

Prior to the advent of the new bilirubin monitoring device (Bilitec), the majority of human evidence for the role of DGOR in oesophageal damage was acquired by prolonged $\mathrm{pH}$ monitoring using $\mathrm{pH}>7$ ("alkaline reflux") as a marker of DGOR..$^{9-11}$ However, the measurement of oesophageal $\mathrm{pH}>7$ as a marker of DGOR is confounded by several problems. Precautions must be taken to use glass electrodes, dietary restriction of food with $\mathrm{pH}<7$, inspection of patients with periodontal disease, and dilatation of strictures to avoid pooling of saliva. ${ }^{1}$ Furthermore, several studies using triple-probe $\mathrm{pH}$ monitoring placed in the oesophagus, gastric fundus and antrum found that oesophageal exposure to $\mathrm{pH}>7$ does not originate from the stomach but rather was due to saliva or bicarbonate production by the oesophageal submucosal glands. ${ }^{12}{ }^{13}$ Additionally, recent studies ${ }^{14}{ }^{15}$ with the Bilitec show no correlation between DGOR and "alkaline reflux". Using simultaneous Bilitec and oesophageal $\mathrm{pH}$ monitoring, Champion and colleagues ${ }^{14}$ found a graded increase in acid and DGOR from controls to patients with oesophagitis with the highest value in patients with Barrett's oesophagus. Although DGOR had a strong correlation with acid reflux $(r=0.78)$, it had a poor correlation with $\mathrm{pH}>7(r=$ $0.06)$. We reported similar findings ${ }^{15}$ in patients with complicated and uncomplicated Barrett's oesophagus. Therefore, these studies suggest that $\mathrm{pH}>7$ - "alkaline reflux"-is a poor marker for oesophageal exposure to duodenal contents and should not be used to assess DGOR.

One of the earliest methods used to evaluate DGOR was the aspiration of gastric or oesophageal contents with fluid analysis for bile acids. The results from the gastric aspiration studies are conflicting. Kaye and Showalter ${ }^{16}$ found no significant difference between fasting gastric bile acid concentrations of patients with oesophagitis compared with controls. Similarly, studies by Gillen and colleagues ${ }^{17}$ found no difference in the fasting bile acid concentrations of patients with complicated or uncomplicated Barrett's oesophagus compared with normal controls. However, we ${ }^{18}$ found that fasting bile acid concentrations were higher in patients with complicated Barrett's oesophagus compared with those with uncomplicated disease, with both being higher than in patients with GORD and controls. A limitation of gastric aspiration studies is the presumption that the presence of bile acids in the stomach is a good indicator of oesophageal exposure to duodenal contents and therefore DGOR. However, only half of DGOR episodes into the antrum reach the fundus of the stomach, and then all that is present in the fundus may not reflux into the oesophagus. ${ }^{19}$

Similarly to studies of gastric aspirates, studies with oesophageal aspiration techniques in humans show conflicting results regarding the role of DGOR in oesophageal mucosal injury. On the one hand Mittal and colleagues ${ }^{20}$ found no bile acids in either the fasting or postprandial oesophageal aspirates of patients with GORD. On the other, Gotley and colleagues ${ }^{21}$ studied 45 patients with oesophagitis and 10 controls using continuous collection of oesophageal aspiration over 16 hours and found increased amounts of conjugated bile acids, measured by high performance liquid chromatography (HPLC), in the majority $(87 \%)$ of aspirates. Most bile acid reflux in this study occurred at night with $7 \%$ of samples having bile acid concentrations above $1.0 \mathrm{mmol} / \mathrm{l}$, the usually toxic concentration producing oesophageal mucosal damage. However, a later study by the same group ${ }^{22}$ found that the oesophageal aspirates of patients with oesophagitis only rarely (2\%) had conjugated bile acid concentrations high enough $(>1.0$ $\mathrm{mmol} / \mathrm{l}$ ) to cause oesophageal mucosal damage. Additionally, they found no unconjugated bile acids or trypsin in the aspirates, whereas acid and pepsin was found in almost all specimens. Thus, supporting the animal studies, they concluded that reflux oesophagitis was caused by acid and 
pepsin with bile acids and trypsin having insignificant roles. Similarly, a recent study by Kauer and colleagues ${ }^{23}$ in 43 normal subjects and 37 patients with GORD found higher concentrations of glycocholic and glycochenodeoxycholic bile acids in the oesophageal aspirates of patients with GORD. However, only two of 37 patients had bile acid concentrations above $1.0 \mathrm{mmol} / 1$.

In this issue (see page 598), Nehra et al used a new oesophageal aspiration technique in an attempt to clarify further the role of DGOR in patients with acid reflux disease. They studied 10 asymptomatic subjects and 30 patients with symptoms of GORD including 10 patients with Barrett's oesophagus. All patients underwent 15 hour continuous oesophageal aspiration with simultaneous $\mathrm{pH}$ monitoring. Using a newly modified HPLC technique, they found significantly $(p<0.05)$ higher concentrations of bile acids in patients with oesophagitis $(124 \mu \mathrm{mol} / \mathrm{l})$ and Barrett's oesophagus $(181 \mu \mathrm{mol} / \mathrm{l})$ than controls $(0 \mu \mathrm{mol} / 1)$. Furthermore, they showed that the predominant bile acids detected were cholic, taurocholic and glycocholic acids. Importantly, they were able to correlate the degree of bile acid reflux with the $\mathrm{pH}$ of the refluxate, finding a temporal relation between acid and taurine conjugated bile acid reflux in the distal oesophagus of patients with GORD ( $r=$ $0.58, \mathrm{p}=0.009)$. This finding confirms our recent report ${ }^{18}$ in which we found that the most $(70-90 \%)$ DGOR measured by the Bilitec in patients with GORD or Barrett's oesophagus occurs in an acidic $(\mathrm{pH}<4)$ milieu, suggesting that acid and DGOR may both be important in causing oesophageal mucosal damage. Furthermore, we ${ }^{18}$ showed that acid and DGOR occur simultaneously more commonly in patients with Barrett's oesophagus (90-100\%) than patients with GORD but without Barrett's oesophagus (50-80\%), suggesting an important synergistic role for acid and DGOR in patients with Barrett's oesophagus. Additionally, the study by Nehra et al confirmed the results from animal studies showing that the taurine conjugated bile acids may be the important bile constituents responsible for oesophageal damage in an acidic milieu.

However, several important points discussed by these authors require further clarification. Nehra et al found that up to $20 \%$ of their patients with GORD have DGOR without acid reflux. Therefore, they concluded that DGOR without acid reflux is damaging to the oesophageal mucosa, and "explains why $15-20 \%$ of patients with GORD fail to respond to acid suppression therapy." However, this conclusion is beyond the scope of their study and is not supported by other reports. We ${ }^{18}$ did not find a significant degree of DGOR without acid reflux in patients with GORD or Barrett's oesophagus. Furthermore, studies show that nearly all patients with GORD and oesophagitis can be treated successfully with adequate acid suppression. $^{2425}$ The $15-20 \%$ treatment failure rate reported in the literature is usually the result of inadequate acid suppression ${ }^{25}$ in drug studies using fixed doses of proton pump inhibitors (PPIs) and is not a function of bile induced oesophageal damage. Furthermore, several studies show that acid suppression with omeprazole reduces oesophageal exposure to acid reflux and also decreases DGOR, most likely by reducing the gastric volume available to reflux into the oesophagus. ${ }^{14}{ }^{26}$ Outside isolated case reports, there is currently little convincing human evidence for the damaging potential of DGOR without acid reflux. For example, studies by Sears and colleagues ${ }^{27}$ in patients with a partial gastrectomy who had prolonged and significant DGOR measured by the Bilitec found that only the patients with concomitant acid reflux had oesophagitis. In this study, the group of patients with DGOR without acid reflux had upper gastroinestinal symptoms (regurgitation, bloating, nausea, and vomiting) but did not have any signs of oesophageal mucosal damage, suggesting that DGOR without acid reflux may cause oesophageal symptoms but does not frequently cause oesophagitis in humans. The symptoms in these patients were successfully treated in $70 \%$ of cases using high dose propulsid $(20 \mathrm{mg}$ po qid). ${ }^{28}$ In those unresponsive to this medical therapy, surgical intervention (Roux-en-Y diversion) can result in adequate symptom relief.

Therefore, currently the literature suggests that both acid and DGOR in patients with GORD and an intact stomach may be important in causing oesophageal mucosal damage. Based on the currently available data, there is no evidence for the damaging potential of DGOR alone without acid reflux in the human oesophagus. Additionally, studies now show that, in an acidic refluxate, taurine conjugated bile acids are the important human bile constituents causing oesophageal damage. In this group of patients, acid suppression with PPIs can reduce both oesophageal acid exposure and DGOR. The reduction in DGOR is most likely related to the reduction in gastric volume secondary to inhibition of acid release by the gastric PPIs. Additionally, conjugated bile acids are precipitated out of solution and pepsin is inactivated at the higher intragastric and intra-oesophageal $\mathrm{pH}$ environments created by the PPIs. Therefore, medical treatment with PPIs or antireflux surgery seems to offer equal protection against the damaging effects of both acid and DGOR in patients with complicated GORD or Barrett's oesophagus.

M F VAEZI

J E RICHTER

Centre for Swallowing and Oesophageal Diseases,

Department of Gastroenterology,

Cleveland Clinic Foundation,

9500 Euclid Avenue,

Cleveland, OH 44195, USA

1 Vaezi MF, Singh S, Richter JE. Role of acid and duodenogastric reflux in esophageal mucosal injury: a review of animal and human studies. Gastroenterology 1995;108:1897-907.

2 Goldberg HI, Dodds WJ, Gee S, et al. Role of acid and pepsin in acute experimental esophagitis. Gastroenterology 1969;56:223-30.

3 Iascone C, DeMeester TR, Little AG, et al. Barrett's esophagus: functional abnormalities, malignant degeneration, and surgical management. Arch Surg 1983;118:543-9.

4 Stein HJ, Hoeft S, DeMeester TR. Reflux and motility pattern in Barrett's esophagus. Dis Esoph 1992;5:21-8.

5 DeMeester TR, Wernly JA, Little AG, et al. Technique, indications and clinical use of 24-hour esophageal pH monitoring. 7 Thorac Cardiovasc Surg 1980;79:656-70.

6 Bremner RM, Crookes PF, DeMeester TR, et al. Concentration of refluxed acid and esophageal mucosal injury. Am ₹ Surg 1992;164:522-7.

7 Harmon JW, Johnson LF, Maydonovitch CL. Effects of acid and bile salts on the rabbit esophageal mucosa. Dig Dis Sci 1981;26:65-72.

8 Kivilaakso E, Fromm D, Silen W. Effect of bile salts and related compounds on isolated esophageal mucosa. Surgery 1980;87:280-5.

9 Pellegrini CA, DeMeester TR, Wernly JA, et al. Alkaline gastroesophageal reflux. Am $\mathcal{F}$ Surg 1978;75:177-84.

10 Attwood SEA, DeMeester TR, Bremner CG, et al. Alkaline gastroesophageal reflux: implications in the development of complications in Barrett's columnar-lined lower esophagus. Surgery 1989;106:764-76.

11 Attwood SEA, Ball CS, Barlow AP, et al. Role of intragastric and intra-oesophageal alkalinisation in the genesis of complications in Barrett's columnar-lined lower oesophagus. Gut 1993;34:11-15.

12 Singh S, Bradley LA, Richter JE. Determinants of oesophageal "alkaline" $\mathrm{pH}$ environment in controls and patients with gastro-oesophageal reflux disease. Gut 1993;34:309-16.

13 Devault KR, Georgeson S, Castell DO. Salivary stimulation mimics esophageal exposure to refluxed duodenal contents. Am $\mathcal{f}$ Gastroenterol geal exposure to

14 Champion G, Richter JE, Vaezi MF, et al. Duodenogastroesophageal reflux: relationship to $\mathrm{pH}$ and importance in Barrett's esophagus. Gastroenterology 1994;107:747-54

15 Vaezi MF, Richter JE. Synergism of acid and duodenogastroesophageal reflux in complicated Barrett's esophagus. Surgery 1994;117:699-704.

16 Kaye MD, Showalter JP. Pyloric incompetence in patients with symptomatic gastroesophageal reflux. f Lab Clin Med 1974;83:198-206.

17 Gillen P, Keeling P, Byrne PJ, et al. Importance of duodenogastric reflux in the pathogenesis of Barrett's oesophagus. BrF Surg 1988;75:540-3.

18 Vaezi MF, Richter JE. Role of acid and duodenogastroesophageal reflux in gastroesophageal reflux disease. Gastroenterology 1996;111:1192-9.

19 Mattioli S, Pilotti V, Felice V, et al. Ambulatory 24-hour $\mathrm{pH}$ monitoring of the esophagus, fundus and antrum. Dig Dis Sci 1990;35:929-38.

20 Mittal RK, Reuben A, Whitney JO, et al. Do bile acids reflux into the esophagus? A study in normal subjects and patients with GERD. Gastroenesophagus? A study in
terology $1987 ; 92: 371-5$

21 Gotley DC, Morgan AP, Cooper MJ. Bile acid concentrations in the refluxate of patients with reflux oesophagitis. Br F Surg 1988;75:587-90. 
22 Gotley DC, Morgan AP, Ball D, et al. Composition of gastro-oesophageal refluxate. Gut 1991;32:1093-9.

23 Kauer WKH, Peters JH, DeMeester TR, et al. Composition and concentration of bile acid reflux into the esophagus of patients with gastroesophageal reflux disease. Surgery 1997;122:874-81.

24 Holloway RH, Dent J, Narielvala F, et al. Relation between oesophageal acid exposure and healing with omeprazole in patients with severe reflux oesophagitis. Gut 1996;38:649-54.

25 Hendel J, Hendel L, Hage E, et al. Monitoring of omeprazole treatment in gastroesophageal reflux disease. Eur f Gastroenterol Hepatol 1996;8:417-20. 26 Marshall REK, Anggiansah A, Owen WA, et al. The relation between acid and bile reflux and symptoms in gastro-oesophageal reflux disease. Gut 1997;40:182-7.

27 Sears RJ, Champion G, Richter JE. Characteristics of partial gastrectomy (PG) patients with esophageal symptoms of duodenogastric reflux. Am $\mathcal{F}$ Gastroenterol 1995;90:211-15.

28 Vaezi MF, Sears R, Richter JE. Placebo-controlled trial of cisapride in postgastrectomy patients with duodenogastroesophageal reflux. Dig Dis Sci 1996;41:754-63.

See article on page 625

\section{Is mycophenolate mofetil a new alternative in the treatment of inflammatory bowel disease?}

As knowledge of the overactive immune response becomes clearer, the treatment of inflammatory bowel disease (IBD) continues to evolve rapidly from the use of antiinflammatory agents to the use of immunomodulatory agents. A wide variety of medications have been shown to be effective in both uncontrolled and controlled trials in either or both ulcerative colitis and Crohn's disease. ${ }^{1}$ These include 6-mercaptopurine (6-MP), azathioprine, methotrexate, cyclosporine, ${ }^{2}$ tacrolimus, interleukin 10, ISIS 2302, and infliximab (a monoclonal antibody against tumour necrosis factor $\alpha$ ).

In this issue (see page 625) a randomised trial reports that treatment with mycofenolate mofetil (MMF) plus steroids was as effective as the combination of azathioprine plus steroids in obtaining a clinical response in chronic active Crohn's disease. The study further suggests that onset of remission was more rapid in those patients with highly active disease and that there were few adverse side effects.

6-Mercaptopurine and its parent drug azathioprine are currently the agents of choice for the treatment of Crohn's disease (and in my experience, for ulcerative colitis as well). Several enzyme systems convert 6-MP to either inactive metabolites or to the 6-thioguanine nucleotides which are purine antagonists and inhibit synthesis of protein, RNA and DNA.

Although 6-MP and azathioprine are effective in the treatment of Crohn's disease, there was an unfortunate delay in the acceptance of the efficacy of these agents because of the faulty design in the National Cooperative Crohn's Disease Study. ${ }^{3}$ This trial studied three agents: steroids, sulfasalazine and azathioprine. The 17 week design failed to account for the slow onset of action of azathioprine (mean of 3.1 months) and missed potential late responders. However, it has become clear from multiple trials and a meta-analysis ${ }^{4}$ that azathioprine/6-MP are effective both in treating active Crohn's disease and in maintaining remission. Complete closure of fistula is observed in about $30 \%$ of patients with improvement in another $25 \%$, whereas consistent response of fistula has not been observed with either 5-aminosalicylic acid agents or steroids. Although the role of combined anti-metabolites and steroids has been controversial because of limited data, the meta-analysis suggests this combination may lead to a higher response rate, with the requirement for less steroids in the long term. Steroid sparing has been confirmed in most studies as well as in the meta-analysis. As regards toxicity, the long term use of azathioprine/6-MP has not been associated with an increase in the development of carcinomas or lymphomas. Several reports in patients with IBD, other immune disorders and transplant patients have also shown no problem with the use of these agents in pregnancy.

Although there are few controlled trials, several uncontrolled studies have shown that azathioprine/6-MP have a similar efficacy in ulcerative colitis. The response rate in the largest series has shown complete clinical remission in over $60 \%$ and a partial response in $24 \%$ of patients. ${ }^{5}$ A single controlled trial has also demonstrated maintenance of remission with azathioprine compared with placebo. Several smaller series have shown that azathioprine/6-MP can maintain remission in severe steroid refractory ulcerative colitis once remission has been induced by cyclosporine. Some clinicians have failed to recognise that early institution of azathioprine/6-MP will significantly reduce the need for subsequent colectomy. A multicentre placebo controlled trial is starting in this year to evaluate the maintenance of efficacy of azathioprine after a severe attack of ulcerative colitis.

With such a clear record of efficacy for azathioprine/6$\mathrm{MP}$, how should we now evaluate the role of MMF in the treatment of Crohn's disease (and possibly ulcerative colitis)? $\mathrm{MMF}$ is a non-competitive selective and reversible inhibitor of inosine monophosphate dehydrogenase (IMP). ${ }^{6}$ Inhibition of IMP depletes guanosine nucleotides in $\mathrm{B}$ and $\mathrm{T}$ lymphocytes. As it inhibits the de novo pathway of purine synthesis that is used differentially by lymphocytes, it might be predicted to have a greater immunosuppressive activity than azathioprine, especially in relation to toxicity.

There have been three randomised placebo controlled trials in transplant patients comparing MMF with either placebo or azathioprine in combination therapy with cyclosporine and steroids. Acute rejection was significantly reduced with MMF compared with either placebo or azathioprine. However, there were no differences between the groups at six and 12 months in terms of graft loss or death. Therefore, significant long term data are limited.

Toxicity of MMF is dose related, with gastrointestinal disturbances (in which diarrhoea and vomiting are most prominent) as well as leucopenia and infectious events. Data on malignancies are limited, but results of initial studies seem to be similar to those seen with azathioprine. The drug is teratogenic in animals and patients are currently advised to avoid pregnancy.

In light of the this information, how should this initial report of the efficacy of MMF in the treatment of chronic active Crohn's disease be evaluated and what recommendations should be made? Although the study was performed well, it has several flaws. Firstly, neither patients nor investigators were blinded as to which medication they were receiving. All patients were said to have chronically active disease, but the severity of a similar degree is questionable as several patients entered into the trial had Crohn's disease activity indexes (CDAI) under 200. A CDAI of this level would constitute mild disease. Secondly, as $5 \mathrm{mg}$ prednisolone was given as maintenance treatment we cannot evaluate complete steroid sparing. I have always felt that complete steroid withdrawal is essential when 
evaluating the efficacy of any drug in the treatment of Crohn's disease.

If future trials confirm a more rapid short term response in severe disease, it would be a strong indication for the use of MMF. However, before MMF can be considered superior to current treatment, controlled trials will be required to show complete steroid sparing, long term efficacy as a maintenance agent, and less toxicity than azathioprine/ 6-MP. For the moment, other than being studied in a controlled trial, I agree with the authors that treatment with MMF should be strongly considered in chronically ill patients with IBD who are either allergic to or have not responded to azathioprine/6-MP.

D H PRESENT

Clinical Professor of Medicine
Mount Sinai Medical Centre,

12 East 86th Street

New York,

NY 10028-0517, USA

1 Hanauer SB. Drug therapy-inflammatory bowel disease. $N$ Engl f Med 1996;334:841-8.

2 Sandborn WJ. A review of immune modifier therapy for inflammatory bowel disease: azathioprine, 6-mercaptopurine, cyclosporine and methotrexate. Am $\mathcal{F}$ Gastroenterol 1996;91:423-33.

3 Present DH. 6-mercaptopurine and other immunosuppressive agents in the treatment of Crohn's disease and ulcerative colitis. Gastroenterol Clin North Am 1989;18:57-71.

4 Pearson DC, May GR, Fick GH, et al. Azathioprine and 6-mercaptopurine in Crohn's disease - a meta-analysis. Ann Intern Med 1995;122:132-42.

5 George J, Present DH, Pou R, et al. The long term outcome of ulcerative colitis treated with 6-mercaptopurine. Am $\mathcal{F}$ Gastroenterol 1996;91:171114

6 Lipsky JJ. Drug profile-mycophenolate mofetil. Lancet 1996;348:1357-9.

\section{Beans means lectins}

Lectins are proteins or glycoproteins of non-immune origin that bind specifically to carbohydrates. They usually, and arguably by definition, have at least two binding sites per molecule and tend to agglutinate cells to which they bind. They are ubiquitous in living matter, whether of plant or animal origin. ${ }^{1}$ Animal lectins include the selectins which are responsible for leucocyte-endothelial interactions, the hepatocyte galactose binding lectin responsible for removing aging, desialylated, glycoproteins from circulation (the asialoglycoprotein receptor), the circulating mannose binding lectin which functions as a complement protein, and the intracellular galectins (galactose binding lectins) whose natural functions have yet to be determined. Microbial lectins include the adhesins that are essential for the pathogenicity of many enteric organisms. Plant lectins are particularly plentiful in seeds and nuts. They are typically globular proteins which are highly resistant to digestion by mammalian enzymes and survive passage though the digestive tract. Their functions in the plant are unclear but probably include growth promoting and antifungal effects. Lectins usually have an effect on the cells to which they bind. Mitogenic functions have long been recognised-for example, for concanavalin A and phytohaemagglutinin. Although the effects of toxic lectins, such as phytohaemagglutinin (red kidney bean lectin) in undercooked chilli con carne, on the gut are well recognised, the interaction between non-toxic dietary lectins and the intestine has been relatively little studied until recently.

In this issue (see page 709), Jordinson and colleagues report that the broad bean lectin inhibits proliferation without apparent cytotoxicity and stimulates differentiation and protein synthesis. This is an unusual and intriguing combination of effects. As is currently the case for most of the known lectin effects, the mechanism is unclear but evidence is presented that the effect on differentiation is related to the adhesion molecule ep-CAM. The lectin is similar in some respects to the non-toxic antiproliferative lectin in the common edible mushroom (Agaricus bisporus).$^{2}$ We have recently found that this lectin becomes internalised and selectively blocks nuclear-localisingsequence-dependent nuclear protein import. ${ }^{3}$ It differs from the broad bean lectin however in that it inhibits rather than stimulates protein synthesis. Care needs to be taken however not to extrapolate too far from results in one cell line to another and particularly when extrapolating from a malignant cell line to a whole animal. It is notable that the stimulation of differentiation is only seen in LS174T and not in HT29 or SW 1222 cells. LS174T, unlike HT29, tend to form well differentiated goblet cells in confluent culture. ${ }^{4}$ Further studies are needed to determine which cell surface glycoproteins bind the lectin, remembering that quite different glycoproteins may express the same carbohydrate structure and it may be just one of these glycoprotein-lectin interactions that is responsible for initiating the differentiation effect. It will then be important to determine whether this broad bean lectin binding glycoprotein is present in the normal or diseased human intestine.

Jordinson and colleagues point out that lectins are plentiful in fruit and vegetables yet intake of these foods is protective against colon cancer, implying that this makes unlikely any connection between pro-proliferative plant lectins and colon cancer. However, this incompletely represents our lectin-galactose hypothesis for diet and colon cancer. ${ }^{5}$ The evidence that peanut ingestion stimulates rectal mucosal proliferation in individuals who express galactose on their mucosal glycoproteins ${ }^{6}$ we take as proof of concept of the principle that important functional interactions are likely to occur between intraluminal lectins and the increased galactose expressed by mucosal glycoproteins in colon cancer and premalignant disease, ${ }^{7}$ rather than proof that dietary galactose binding lectins will prove to be a major cause of colon cancer. We have pointed out that many of the intraluminal lectins will be of microbial origin and that the role of dietary galactose, which will competitively bind and therefore inhibit many of these lectins, may be a more important mechanism to explain the protective effect of fruit and vegetable fibre against colon cancer. A recent case control study of diet and colon cancer in Liverpool supports the protective effect of dietary galactose. ${ }^{8}$

It is not possible to predict what effects the broad bean lectin will have on the intact human intestine and Jordinson et al's conclusion that the broad bean lectin taken in the diet "may slow progression of colon cancer" is interesting but highly speculative. There are many unpredictable factors which include possible interactions between the lectin and dietary carbohydrates, interaction between the lectin and intestinal bacteria, the ability of the lectin to resist heat and digestion, and the possibility that the lectin might have effects on cells other than colon epithelial cells. Pusztai and colleagues have shown that many of the toxic effects of some lectins are dependent on their interaction with the intestinal flora $^{9}$ and we have shown that dietary lectins may become internalised and circulate intact in the peripheral blood. ${ }^{10}$ 
The whole field of epithelial glycobiology and its implications for interaction between the mucosa and intraluminal lectins of dietary or microbial origin is fascinating and ripe for further study. Many of the glycosylation abnormalities found in colon cancer have been shown to correlate with invasive potential and ultimate prognosis. Some of the glycosylation changes are likely to be under genetic control-that is, as mucosally expressed blood group carbohydrate antigens. Much remains to be discovered about the nature of lectin-epithelial cell interactions and their implications for the functional importance of the regulation of glycosylation on cell surface and intracellular epithelial glycoproteins. Some of the plant lectins, such as the broad bean and mushroom lectins, may prove very useful tools in helping to identify key cellular glycoproteins involved in the regulation of proliferation and differentiation and its alteration in malignant disease.

Department of Medicine,

J M RHODES

University of Liverpool,

Liverpool L69 3GA, UK

email rhodesjm@liverpool.ac.uk
1 Pusztai A, Bardocz S (eds). Lectins. Biomedical perspectives. London: Taylor and Francis, 1995.

2 Yu L, Fernig DG, Smith JA, et al. Reversible inhibition of proliferation of epithelial cell lines by Agaricus bisporus (edible mushroom) lectin. Cancer Res 1993;53:4627-32.

3 Yu L-G, Fernig DG, White MRH, et al. Edible mushroom (Agaricus bisporus) lectin, which reversibly inhibits epithelial cell proliferation, blocks NLS-dependent nuclear protein import. F Biol Chem (in press).

4 Rutzky LP, Tomita JT, Calenoff MA, et al. Human colon adenocarcinoma cells. III. In vitro organoid expression and carcinoembryonic antigen kinetics in hollow fiber culture. $\mathcal{F}$ Natl Cancer Inst 1979;63:893-902.

5 Rhodes JM. Unifying hypothesis for inflammatory bowel disease and related colon cancer: sticking the pieces together with sugar. Lancet 1996;347:40-4.

6 Ryder SD, Jacyna MR, Levi AJ, et al. Eating peanuts increases rectal proliferation in individuals with mucosal expression of peanut lectin receptor. eration in individuals with mucos
Gastroenterology 1998;114:44-9.

7 Campbell BJ, Hounsell E, Finnie IA, et al. Direct demonstration of increased expression of Thomsen-Friedenreich antigen (Galp1-3GalNAc) by mucus in colon cancer and inflammatory bowel disease. 7 Clin Invest 1995;95:571-6.

8 Evans RC, Ashby D, Hackett A, et al. Consumption of peanuts, which contain a galactose-binding lectin, associates with increased risk for colorectal cancer whereas high non-starch polysaccharide galactose intake associates with reduced risk. Gut 1997;41(suppl 3):A124.

with reduced risk. Gut 1997;41 (suppl 3):A124.
9 Pusztai A, Grant G, Spencer RJ, et al. Kidney bean lectin-induced Escherichia coli overgrowth in the small intestine is blocked by GNA, a mannose-specific lectin. f Appl Bacteriol 1993;75:360-8.

10 Wang Q, Yu L-G, Campbell BJ, et al. Identification of intact peanut lectin in peripheral venous blood. Lancet 1998;352:1831-2.

\section{PBC and the gut: the villi atrophy, the plot thickens}

An association between primary biliary cirrhosis (PBC) and coeliac disease now seems well established. Since the original description by Logan and colleagues, ${ }^{1}$ there have been several reports indicating an association and these have been strengthened by larger epidemiological studies. ${ }^{2-11}$ In this issue (see page 736), Sørensen and colleagues publish their analysis of the prevalence of PBC in two populations of patients with coeliac disease, and confirm the association with a standardised incidence ratio of PBC in patients with coeliac disease of about 26. That separate evaluation of the two populations, Danes and Swedes (two countries with very different prevalences of coeliac disease), gave similar incidence ratios is strong evidence for a real association. If for no other reason, this paper deserves widespread recognition because it highlights the clinical insights that come from accurate and complete national morbidity and mortality registers. We in the United Kingdom would do well to learn from the Scandinavian example.

Last year Kingham and Parker showed, in a well defined population in Swansea, South Wales, that the prevalence of PBC in those followed for coeliac disease was 3\% and the prevalence of coeliac disease in those with PBC was $6 \% .{ }^{11}$ In Northern Ireland, routine screening of 67 patients with PBC showed that $11 \%$ had IgA endomysial antibodies and of these, four agreed to have a duodenal biopsy which showed villous atrophy, giving a minimum prevalence rate of $7 \% .^{8}$ These figures are considerably higher than those given by Sorensen et al who found 24 cases of PBC (0.3\%) among 8631 coeliac patients followed in the Danish and Swedish populations. This 10-fold discrepancy between the Welsh and Scandinavian data may in part be due to real differences and in part to different methods. In Wales, all patients with PBC were screened for coeliac disease if they had signs or symptoms of malabsorption (steatorrhoea, haematinic deficiency, a positive family history or antigliadin antibodies present in serum); coeliac patients were screened for PBC if they had abnormal biochemistry. It is not clear in Sorensen et al's paper whether all patients with coeliac disease were systematically evaluated for PBC. That neither series prospectively measured antimitochondrial antibodies in those with coeliac disease suggests that both figures are likely to be underestimates.

Do these observations mean those patients with either PBC or coeliac disease should be screened for the other? The answer is probably yes. Certainly, I believe that patients with PBC should be screened for coeliac disease: as is evident from the clinical studies some cases of coeliac disease were recognised only when specifically looked for. Moreover, the clinical and biochemical consequences of undiagnosed coeliac disease may be wrongly attributed to progression of the underlying liver disease. How best to screen is uncertain. Floreani and colleagues ${ }^{12}$ found that antigliadin antibodies were present in up to $7 \%$ of patients with PBC but low antibody titres were more likely to be secondary to liver damage and only high titre antibodies were associated with coeliac disease. Antiendomysial antibodies are much more specific for coeliac disease. ${ }^{13}$ Whether a duodenal biopsy is necessary to confirm the diagnosis is more controversial and indeed a duodenal biopsy may be contraindicated in those rare instances of PBC where the thrombocytopenia or prolonged clotting (uncorrectable by parenteral vitamin $\mathrm{K}$ ) makes the chance of bleeding too high. My own prejudice that duodenal biopsy should be undertaken to confirm or refute the diagnosis is based purely on anecdote. We recently investigated one woman with PBC and high titres of antiendomysial antibodies in whom repeated duodenal biopsies showed no evidence of coeliac disease. Although there are no data on the cost-effectiveness of screening patients with PBC for coeliac disease, screening is certainly justified clinically. Recently, two patients with PBC have been referred to our liver unit for transplantation because of deteriorating liver tests, lethargy and diarrhoea; coeliac disease was diagnosed and treated with a consequent improvement so that transplantation was no longer needed.

Screening patients with coeliac disease for PBC is more problematic, especially if they are asymptomatic. There are many causes of abnormal liver tests in these patients. It is easy and relatively cheap to screen for PBC as antimitochondrial antibodies can be detected in over $95 \%$ of cases, but the benefits of diagnosing a progressive disease that 
Table 1 Similarities and dissimilarities between primary biliary cirrhosis $(P B C)$ and coeliac disease (see text for references)

\begin{tabular}{|c|c|c|}
\hline & Coeliac disease & $P B C$ \\
\hline Sex distribution (F:M) & $3: 2$ & $9: 1$ \\
\hline HLA associations & $\begin{array}{l}\text { DR3DQ2; DR5DR7; } \\
\text { DR4DQ8 }\end{array}$ & DR8 \\
\hline $\begin{array}{l}\text { Incidence in first degree } \\
\text { relatives }\end{array}$ & $10-20 \%$ & $3-4 \%$ \\
\hline Disease associations & $\begin{array}{l}\text { Dermatitis herpetiformis; } \\
\text { diabetes mellitus; Down's } \\
\text { syndrome }\end{array}$ & $\begin{array}{l}\text { Thyroid disease; other } \\
\text { autoimmune diseases }\end{array}$ \\
\hline Seen in children & Yes & No \\
\hline
\end{tabular}

cannot be cured is uncertain. As yet there is no definitive treatment for PBC. Ursodeoxycholic acid (UDCA) is the only treatment licensed for PBC and although remarkably free of side effects it will not prevent progression even though most evidence suggests it may slow the rate of progression. Clinical studies have concentrated on symptomatic patients with PBC so it is not yet established whether UDCA has a beneficial effect when given in early PBC. ${ }^{14}$ Thus, for the asymptomatic patient, little may be gained by diagnosing an untreatable condition and giving the patient the burden of another problem. Conversely, there may be advantages for the patient in making the diagnosis. Symptoms and signs of PBC, such as diarrhoea, upper abdominal pain, lethargy, or abnormalities in liver tests, may be inappropriately attributed to coeliac disease or its complications. Obviously, if the patient is experiencing symptoms of PBC, clarifying the diagnosis will allow for a more rational approach to management.

One of the intriguing questions raised by these observations is why there should be an association between the two diseases. There are several features which the two conditions have in common (table 1) including an association with autoimmune diseases, familial occurrence and an HLA association. ${ }^{1516}$ However, closer examination suggests that the differences are greater than the similarities: both conditions have a weak HLA association, but with different phenotypes. The primary HLA association of coeliac disease is with the HLA genotypes DQA $1{ }^{\star} 0501$ and DQB $1{ }^{\star} 0201$ which encode the DQ2 heterodimer which is in linkage equilibrium with the extended HLA haplotype HLA A1,B8,DR3,DQ2. This may account for part of the association of coeliac disease with other autoimmune diseases. ${ }^{17}$ No such clear HLA association with A1,B8,DR3 exists for PBC. ${ }^{18}{ }^{19}$ There may be a correlation between the duration of exposure to gluten and the risk of developing autoimmune disease..$^{20}$ It has been suggested that the generation of the autoantibodies to gliadin and endomysial antigens is an autoimmune one. ${ }^{16}$ In both diseases there is a female preponderance but this is much more noticeable in PBC. The associated autoimmune diseases differ: of the autoimmune diseases associated with coeliac disease, dermatitis herpetiformis is not closely associated with PBC and diabetes mellitus rarely so. ${ }^{15}$ Conversely, thyroid disease, seen in up to $20 \%$ of patients with PBC, is less common in those with coeliac disease. ${ }^{21}$ Although diabetes mellitus is associated both with PBC and coeliac disease, only in coeliac disease is the association with insulin dependent diabetes. Again, although both conditions do have an increased incidence in first degree members, the figure varies greatly. PBC is associated with hypergammaglobulinaemia and coeliac disease with selective IgA deficiency. More tellingly, however, coeliac disease, which may be diagnosed at any age, is more common in children whereas PBC has not been diagnosed in children. ${ }^{14}{ }^{15}$

Despite these differences, it is tempting to use the association to consider whether the pathogenesis of coeliac disease can suggest clues for the pathogenesis of PBC. A simplified pathogenesis of coeliac disease is that gluten, absorbed into the lamina propria, is presented by the dendritic cells to local, sensitised lymphocytes which then stimulate antibody, cytokine and cell mediated enterocyte damage. ${ }^{16}$ Whether PBC is also triggered by an exogenous antigen is far from certain. Bacteria and viruses have been implicated in the pathogenesis of PBC but few are convinced that any definite trigger has been identified, never mind account for the link between PBC and the antimitochondrial antibody. ${ }^{22}$

It may be that there is a congenital or acquired defect of immunoregulation in patients with PBC which also promotes an immune response to gliadin. An alternative explanation, raised by Kingham and Parker, is that IgA may be involved. In epithelial cells ${ }^{11}$ IgA antibodies play a major role in mucosal defences and are potentially powerful participants in inflammatory processes. IgA is transported through epithelial cells, allowing antigens or even viruses to enter these epithelial cells. ${ }^{23} 24$ Although both diseases can be postulated to result from exposure to a foreign antigen with genetic and environmental factors determining the time of presentation and rate of progression, only in coeliac disease is the trigger identified. The trigger in PBC remains elusive.

J NEUBERGER

Liver Unit,

Queen Elizabeth Hospital,

Birmingham B15 2TH, UK

emailj.m.neuberger@bham.ac.uk

1 Logan RFA, Ferguson A, Finlayson ND, et al. Primary biliary cirrhosis and coeliac disease, an association? Lancet 1978;i:230-3.

2 Iliffe GD, Owen DA. An association between primary biliary cirrhosis and jejunal villous atrophy resembling coeliac disease. Dig Dis 1979;24:802-6.

3 Behr W, Barnert J. Adult celiac disease and primary biliary cirrhosis. Am $\mathcal{f}$ Gastroenterol 1986;81:796-9.

4 Olsson I, Kagevi I, Rydberg L. On the concurrence of primary biliary cirrhosis and intestinal villous atrophy. Scand $\mathcal{F}$ Gastroenterol 1982;17:6258

5 Ginn P, Workman D. Primary biliary cirrhosis and adult coeliac disease. West f Med 1992;156:547-9.

6 Lohr M, Lotterer E, Hahn EG, et al. Primary biliary cirrhosis associated with coeliac disease. Eur f Gastroeneterol Hepatol 1994;6:263-7.

7 Niveloni S, Dezi R, Pedreira S, et al. Gluten sensitivity in patients with primary biliary cirrhosis. Am f Gastroenterol 1998;93:404-8.

8 Dickey W, McMillan SA, Callender ME. High prevalence of celiac sprue among patients with primary biliary cirrhosis. F Clin Gastroenterol 1997;25: 328-9.

9 Bardella MT, Quatrini M, Zuin M, et al. Screening patients with celiac disease for primary biliary cirrhosis and vice versa. Am $f$ Gastroenterol 1997;92:1524-6.

10 Glavez C, Garrigues V, Ponce J. Primary biliary cirrhosis and coeliac disease. Eur $\mathcal{F}$ Gastroenterol Hepatol 1994;6:847.

11 Kingham JGC, Parker DR. The association between primary biliary cirrhosis and coeliac disease: a study of relative prevalences. Gut 1998;42:120-2.

12 Floreani A, Chiaramonte $M$, Venturini $R$, et al. Antigliadin antibody classes in chronic liver diseases. Ital f Gastroenterol 1992;24:457-60.

13 Sjoberg K, Lindgren S, Eriksson S. Frequent occurrence of non-specific gliadin antibodies in chronic liver disease. Endomysial but not gliadin antibodies predict coeliac disease in patients with chronic liver disease. Scand $\mathcal{F}$ Gastroenterol 1997;32:1162-7.

14 Neuberger J. Primary biliary cirrhosis. Lancet 1997;350:875-9.

15 Maki M, Collin P. Coeliac disease. Lancet 1997;349:1755-9.

16 Godkin A, Jewell D. The pathogenesis of celiac disease. Gastroenterology 1998;115:206-10.

17 Branski D, Troncone R. Celiac disease: a reappraisal. $f$ Paediatr 1998;133:181-7.

18 Manns M, Kruger M. Immunogenetics of chronic liver diseases. Gastroenterology 1994;106:1676-9.

19 Gregory W, Bassendine M. Genetic factors in primary biliary cirrhosis. $\mathcal{F}$ Hepatol 1994;20:689-92.

20 Ventura A, Maguzza G, Greco L. Autoimmune disorders in coeliac disease: relationship with duration of exposure to gluten [abstract]. $\mathscr{F}$ P $F$ F Pediatr Gastroenterol Nutr 1997;24:463.

21 Metcalf JV, James OFW. Vascular and autoimmune disease "associations" with PBC. The best evidence available [abstract]. Hepatology 1996;24: $168 \mathrm{~A}$.

22 Neuberger J, Thomson R. AMA and PBC-what is the connection? Hepatology 1999;29:271-6.

23 Lamm ME. Interaction of antigens and antibodies at mucosal surfaces. Ann Rev Microbiol 1997;51:311-40.

24 Lamm ME. Current concepts in mucosal immunity. IV. How epithelial transport of IgA antibodies relates to host defence. Am f Physiol 1998;274: G614-17. 